\title{
Influencing Factors and Rules of Behavioral Intention of Cesarean Section in Late Pregnancy
}

\author{
Zhuo Lu ${ }^{1}$, Xian Tang ${ }^{2}$, Jiaxiu Liu ${ }^{2}$ and Xiaoni Zhong ${ }^{2, *}$ \\ ${ }^{1}$ Applied Statistics, Chongqing Medical University, Chongqing, China \\ ${ }^{2}$ School of Public Health and Management, Chongqing Medical University, Chongqing, China
}

*Corresponding author: Xiaoni Zhong, School of Public Health and Management, Chongqing Medical University, Chongqing, China, Tel: +861330-836-8059; E-mail: zhongxiaoni@cqmu.edu.cn

Received: 01 Feb, 2020 | Accepted: 24 Feb, 2020 | Published: 28 Feb, 2020

Citation: Lu Z, Tang X, Liu J, Zhong X (2020) Influencing Factors and Rules of Behavioral Intention of Cesarean Section in Late Pregnancy. J Epidemiol Public Health Rev 5(1): dx.doi.org/10.16966/2471-8211.182

Copyright: (C 2020 Lu Z, et al. This is an open-access article distributed under the terms of the Creative Commons Attribution License, which permits unrestricted use, distribution, and reproduction in any medium, provided the original author and source are credited.

\section{Abstract}

Objective: To explore the psychological and sociological factors that affect women's behavioural intention of cesarean section in late pregnancy, so as to provide theoretical basis for guiding the opinion orientation of key groups, encouraging pregnant women to adopt natural delivery and reducing the rate of cesarean section.

Methods: Based on a longitudinal observational study of women's intention to cesarean section during pregnancy in Chongqing, China, multiple logistic regression was used to explore the influencing factors of women's selective cesarean section.

Result: The results showed that $4.64 \%$ of pregnant women had selective cesarean section. The occupation of pregnant women, exercise in late pregnancy, delivery mode of surrounding parturients, and suggestion of husband/parents/friends were significantly affected the women's choice of mode of delivery. The results of multivariate analysis showed that cesarean section suggested by friends (OR=37.172), elderly parturient aged 30-35 $(O R=6.285)$, and the gestation-parturition history $(O R=3.462)$ were the risky factors associated with the increase of selective cesarean section.

Conclusion: Pregnant women's childbirth related knowledge level, family and friends' suggestions affect pregnant women's choice of childbirth mode. Therefore, it is necessary to improve pregnant women's and their family and friends' childbirth related knowledge level, make more scientific suggestions for pregnant women's choice of childbirth mode, at the same time, strengthen women's attention to the golden age of childbirth.

Keywords: Behavioral intention; Cesarean section; Delivery mode; Opinion orientation; Influencing factors

\section{Introduction}

Cesarean section is an effective method to solve the obstetric dangerous symptoms such as dystocia and malposition, which is also a powerful means to save the lives of both perinatal and newborn [1]. According to the survey conducted in Asia by WHO in 2010, the cesarean section rate in China was $46.2 \%$, ranking first in Asia, which was far higher than $10 \%-15 \%$ advocated by Word Health Organization [2], and the cesarean section rate has also far exceeded the range of simple medical indications. With a lack knowledge of delivery, many pregnant women blindly believe that cesarean section is the best way to ensure the safety of mother and baby $[3,4]$. Moreover, previous studies also found that cesarean section had a negative impact on the second delivery of pregnant women, that the second pregnancy was prone to experience the adverse consequences of uterine rupture, high incidence of placenta previa in mothers, and neonatal dyspnea and low resistance in newborns [5]. Therefore, the choice of delivery mode has a significant impact on the delivery outcome [6].

In recent years, more and more attention has been paid to natural delivery and prenatal health education, but the health education was limited only in the hospital, which means little in promoting the whole society to correctly understand natural delivery through vagina. In addition, pregnant women's choice of delivery mode is easily affected by many external factors among them social factors are the main factors affecting the high rate of cesarean section [7-11]. In addition, with the increase of gestational age, the mental health status of pregnant women, the level of knowledge related to delivery, exercise during pregnancy, and their own physical fitness have also been changed. So gestational period is an important factor affecting the behavioural intention of cesarean section of pregnant women. Therefore, it is of great significance to explore the factors that affect the choice of delivery mode of cesarean section in different gestational periods, in order to reduce the rate of cesarean section with nonmedical indications and guide pregnant women with natural delivery conditions to choose vaginal delivery [12].

\section{Materials and Methods}

\section{Study procedures}

The data of our study were collected from "Study on the Public 
Opinion Propagation Model for Generative Mechanism and Regularity of Cesarean Delivery Behavior", which was initiated by the National Natural Science Foundation of China (Project No.: 71573027). The study has been approved by Ethics Committee of Chongqing Medical University and conducted in Chongqing, a provincial city in China. Participants were recruited from four regions with different economic conditions in Chongqing (Yubei district, Jiangjin district, Dianjiang district and Yunyang district). After entering the group, the nurses of Maternal and Child Health Station who have received unified training were responsible for carrying out questionnaire survey and followup records. According to the inclusion exclusion criteria, pregnant women who built cards in the local maternal and child health station during March 1, 2018 to January 31, 2019 and had regular prenatal examination were followed up for cesarean section intention. Finally, 517 pregnant women were included in this study and subjects who chose caesarean section for medical reasons were not included.

Inclusion criteria: (a) Women with singleton pregnancy; (b) Willingness to sign the informed consent and obey the follow-up arrangement under the guidance.

Exclusion criteria: (a) Women with multiple pregnancy; (b) Women with histories of cesarean section; (c) Women with health problems, such as mental illness; (d) Refuse to sign informed consent.

\section{Study contents}

According to the gestational period of pregnant women, the questionnaire survey is divided into six surveys: first birth examination, early pregnancy, mid pregnancy, late pregnancy, postpartum and delivery records. The data of this manuscript comes from the survey of late pregnancy. The questionnaire includes social demographic characteristics such as age, residence, educational level, working status during pregnancy and monthly per capita household income; general personal condition of pregnant women; prenatal basic information including gestation-parturition history, Body Mass Index (BMI) before pregnancy; Whether to exercise in the late pregnancy, suggestions of family and friends on delivery mode, knowledge level of delivery, pregnant women's independence, family care, social support, mental health status, choice intention of pregnant women's personal delivery mode, delivery mode of surrounding parturients, etc.

\section{Study measurements}

Measurements description: (1) Prenatal stress of pregnant women was assessed by Pregnancy Pressure Scale (PPS), compiled by Zhanghui ZJ, et al. [13]. The scale has been widely applied to related studies in Chinese pregnant women and showed favorable reliability and validity [14], an average score of $>0$ was considered stressful. (2) Prenatal anxiety of pregnant women was measured by Hamilton Anxiety Scale (HAMA), which has been widely used in Chinese pregnant women. A total score $>14$ was considered to be anxiety. (3) The Self-Rating Depression Scale (SDS) was used to evaluated the prenatal depression of pregnant women, when the index was $\geq 0.5$, it was considered to have depression symptoms. (4) Family care was assessed by the family adaptation partnership growth affection and resolve index (APGAR) used in Chinese pregnant women and proven to be valid and reliable $[15,16]$. Higher scores indicate better family care, with $0-3$ for a low level, 4-6 for a moderate level, and 7- 10 for a high level. (5) Social support was evaluated by Social Support Rating Scale (SSRS) compiled by Xiao Shuiyuan in 1986. Scores below 35 indicate low level, 35-45 indicate medium level, and above 45 indicates high level, and the higher the score, the more social support.

\section{Statistical analysis}

The database was established by the EpiData 3.1 software (EpiData Associations, Odense, Denmark), and real-time double entry and logical verification of the data were carried out. Statistical analysis was performed by the SAS 9.4 software (SAS Institute, Cary, NC, USA). Univariate analysis was performed by the $\chi^{2}$ test, and logistic regression model was used in multivariate analysis, with $\mathrm{p}<0.05$ was considered statistically significant.

\section{Results}

\section{Subjects' characteristics}

A total of 517 subjects were included in this study. In the late pregnancy, the pregnant women's selective intention of cesarean section accounted for 24 (4.64\%), natural delivery for 280 (54.16\%), and not yet considered for $213(41.20 \%)$. The mean age of pregnant women was $(25.67 \pm 4.25)$ years, and the mean BMI before pregnancy was $(21.10 \pm 2.68) .33 .27 \%$ of the pregnant women surveyed had junior high school education or below, $28.43 \%$ had senior high school education, and $38.39 \%$ had college education or above.

\section{Expected delivery mode of pregnant women during pregnancy}

In this study, the proportion of selective cesarean section in late follow-up visit was $4.64 \%$ (Table 1 ). There was no significant difference in the incidence of cesarean section as an expected mode of delivery between age, nationality, Residence, educational level, monthly per capita household income, payment mode of medical expenses, number of prenatal education in pregnancy, care of doctors and nurses, independence of pregnant women, family care, prenatal stress, prenatal anxiety, social support, BMI, and gestation-parturition history $(\mathrm{P}>0.05)$. However, in the univariate analysis, significant differences in the choice of expected delivery mode in the late stage of pregnancy were whether to exercise in late pregnancy, delivery mode of surrounding parturients, delivery mode suggested by husband, parents and friends $(\mathrm{P}<0.05)$.

\section{Multivariate logistic regression analysis}

The stepwise regression method was used for variables screening, with each variable visited in each period as the independent variables, whether to choose cesarean section as the dependent variable, and the categorical variable was assigned a dummy variable (Table 2 ). In the late follow-up visit, there were four variables in the model, which were occupation, delivery mode suggested by friends, age and gestationparturition history. Freelance and unemployment status would increase the risk that pregnant women choose cesarean section as their delivery mode. Compared with no suggestion, cesarean section suggested by friends would increase the risk of pregnant women choosing cesarean section as the delivery mode ( $\mathrm{OR}=37.172,95 \% \mathrm{CI}$ : 6.986-197.797). Moreover, younger women with a maternal age $<20$, and older women with age of 30-35 years were inflation factors relative to women's normal reproductive age of 20-29, and the risk of choosing a cesarean section as the delivery mode were 3.098 and 6.285 times than the normal age group respectively. Besides, pregnant women with a gestation-parturition history were more likely to choose cesarean section as the delivery method compared to those without births.

\section{Discussion}

Cesarean section with non-medical indications has a serious impact on the health of mothers and newborns, and controlling the rate of cesarean section with non-medical indications can effectively 
Table 1: Sample characteristics of pregnant women and univariate analysis.

\begin{tabular}{|c|c|c|c|}
\hline Characteristics & Natural delivery/Not sure & Cesarean Section & p-Value \\
\hline & $N(\%)$ & $N(\%)$ & \\
\hline Age & & & $0.0733^{*}$ \\
\hline$<20$ & $11(2.13)$ & $2(0.39)$ & \\
\hline $20-29$ & 403(77.95) & $16(3.09)$ & \\
\hline $30-35$ & 63(12.19) & $6(1.16)$ & \\
\hline$>35$ & $16(3.09)$ & $0(0)$ & \\
\hline Nationality & & & $0.4204^{*}$ \\
\hline Han nationality & 480(92.84) & $24(4.64)$ & \\
\hline Minority nationality & $13(2.51)$ & $0(0)$ & \\
\hline Residence & & & 0.5666 \\
\hline Urban & 296(57.25) & $13(2.51)$ & \\
\hline Rural & $197(38.1)$ & $11(2.13)$ & \\
\hline Educational level & & & 0.8852 \\
\hline Junior high school or below & $163(31.53)$ & $9(1.74)$ & \\
\hline Senior high school & $141(27.27)$ & $6(1.16)$ & \\
\hline College education or above & $189(36.56)$ & $9(1.74)$ & \\
\hline Working status during pregnancy & & & 0.0069 \\
\hline Employment & $145(28.05)$ & $3(0.58)$ & \\
\hline Freelance & $85(16.44)$ & $10(1.93)$ & \\
\hline Housewife/Unemployment & $263(50.87)$ & $11(2.13)$ & \\
\hline Monthly per capita household income & & & 0.9067 \\
\hline$\leq 3000 \mathrm{RMB}$ & $127(24.56)$ & $7(1.35)$ & \\
\hline 3000-5000RMB & $215(41.59)$ & $9(1.74)$ & \\
\hline 5000-10000RMB & $123(23.79)$ & $7(1.35)$ & \\
\hline$>10000 R M B$ & $28(5.42)$ & $1(0.19)$ & \\
\hline Payment mode of medical expenses & & & $0.1395^{*}$ \\
\hline At their own expense & $184(35.59)$ & $14(2.72)$ & \\
\hline Urban medical insurance & $304(58.8)$ & $10(1.95)$ & \\
\hline Rural cooperative medical insurance & $2(0.39)$ & $0(0)$ & \\
\hline Whether to exercise in late pregnancy & & & 0.0343 \\
\hline Yes & $347(67.12)$ & $12(2.32)$ & \\
\hline No & $146(28.24)$ & $12(2.32)$ & \\
\hline Number of prenatal education in pregnancy & & & 0.9788 \\
\hline 0 & $277(53.58)$ & $14(2.72)$ & \\
\hline 5-Jan & $173(33.46)$ & $8(1.55)$ & \\
\hline$>5$ & $43(8.32)$ & $2(0.39)$ & \\
\hline Medical Staff Service & & & 0.4938 \\
\hline Great & $343(66.34)$ & $15(2.90)$ & \\
\hline Moderate & $115(22.24)$ & $8(1.55)$ & \\
\hline Low & $35(6.77)$ & $1(0.19)$ & \\
\hline Delivery mode of surrounding parturients & & & 0.0034 \\
\hline
\end{tabular}

Citation: Lu Z, Tang X, Liu J, Zhong X (2020) Influencing Factors and Rules of Behavioral Intention of Cesarean Section in Late Pregnancy. 


\begin{tabular}{|c|c|c|c|}
\hline Natural delivery mainly & $227(43.91)$ & $5(0.97)$ & \\
\hline Cesarean section mainly & $59(11.41)$ & $8(1.55)$ & \\
\hline The two delivery methods are equal & $207(40.04)$ & $11(2.13)$ & \\
\hline Delivery mode suggested by husband & & & $0.0009 *$ \\
\hline No Suggestion & 107(20.7) & $4(0.77)$ & \\
\hline Natural Delivery & $211(40.81)$ & $7(1.35)$ & \\
\hline Cesarean Section & $12(2.32)$ & $4(0.77)$ & \\
\hline No Specific Suggestion & $163(31.53)$ & $9(1.74)$ & \\
\hline Delivery mode suggested by parents & & & 0.0006 \\
\hline No Suggestion & $84(16.25)$ & $5(0.97)$ & \\
\hline Natural Delivery & $270(52.22)$ & $7(1.35)$ & \\
\hline Cesarean Section & $7(1.35)$ & $4(0.77)$ & \\
\hline No Specific Suggestion & $132(25.53)$ & $8(1.55)$ & \\
\hline Delivery mode suggested by parents in law & & & $0.0719 *$ \\
\hline No Suggestion & $99(19.15)$ & $4(0.77)$ & \\
\hline Natural Delivery & $224(43.33)$ & $8(1.55)$ & \\
\hline Cesarean Section & $6(1.16)$ & $2(0.39)$ & \\
\hline No Specific Suggestion & $164(31.72)$ & $10(1.93)$ & \\
\hline Delivery mode suggested by friends & & & $<0.0001^{*}$ \\
\hline No Suggestion & $98(18.96)$ & $4(0.77)$ & \\
\hline Natural Delivery & $210(40.62)$ & $4(0.77)$ & \\
\hline Cesarean Section & $7(1.35)$ & $8(1.55)$ & \\
\hline No Specific Suggestion & $178(34.43)$ & $8(1.55)$ & \\
\hline Independence of pregnant women & & & $0.6889 *$ \\
\hline Group-Oriented & $18(3.48)$ & $1(0.19)$ & \\
\hline Intermediate State & $470(90.91)$ & $23(4.45)$ & \\
\hline Self-Sufficient & $5(0.97)$ & $0(0)$ & \\
\hline Knowledge level of delivery & & & 0.1310 \\
\hline Low & $95(18.38)$ & $6(1.16)$ & \\
\hline Moderate & $266(51.45)$ & $16(3.09)$ & \\
\hline High & $132(25.53)$ & $2(0.39)$ & \\
\hline Family care & & & 0.8617 \\
\hline Severe family dysfunction & $14(2.71)$ & $1(0.19)$ & \\
\hline Moderate family dysfunction & $108(20.89)$ & $6(1.16)$ & \\
\hline Family functioning well & $371(71.76)$ & $17(3.29)$ & \\
\hline Prenatal stress & & & $0.4745^{*}$ \\
\hline No & $58(11.22)$ & $1(0.19)$ & \\
\hline Slight & $393(76.02)$ & $20(3.87)$ & \\
\hline Moderate & $41(7.93)$ & $3(0.58)$ & \\
\hline Severe & $1(0.19)$ & $0(0)$ & \\
\hline Prenatal anxiety & & & 0.2286 \\
\hline No & $347(67.12)$ & $14(2.72)$ & \\
\hline Moderate & $136(26.31)$ & $9(1.74)$ & \\
\hline Severe & $10(1.93)$ & $1(0.19)$ & \\
\hline
\end{tabular}

Citation: Lu Z, Tang X, Liu J, Zhong X (2020) Influencing Factors and Rules of Behavioral Intention of Cesarean Section in Late Pregnancy. 


\begin{tabular}{|c|c|c|c|}
\hline Prenatal depression & & & $0.5567^{*}$ \\
\hline No & $486(94)$ & $24(4.64)$ & \\
\hline Yes & $7(1.35)$ & $0(0)$ & \\
\hline Social support & & & 0.9385 \\
\hline Low & $106(20.5)$ & $5(0.97)$ & \\
\hline Moderate & $292(56.48)$ & $15(2.90)$ & \\
\hline High & $95(18.38)$ & $4(0.77)$ & \\
\hline BMI & & & $0.5722^{*}$ \\
\hline Thin & $80(15.47)$ & $6(1.16)$ & \\
\hline Normal & $350(67.7)$ & $17(3.29)$ & \\
\hline Overweight & $52(10.06)$ & $1(0.19)$ & \\
\hline Obese & $11(2.13)$ & $0(0)$ & \\
\hline Gestation-parturition history & & & 0.1168 \\
\hline No & $336(64.99)$ & $20(3.87)$ & \\
\hline Yes & $157(30.37)$ & $4(0.77)$ & \\
\hline
\end{tabular}

*Fisher's exact probability test

Bold values indicate statistical significance at $\mathrm{P}<0.05$.

Table 2: Multivariate logistic regression analysis.

\begin{tabular}{|c|c|c|c|c|}
\hline Characteristics & $\beta$ & Wald & P-Value & OR (95\% CI) \\
\hline \multicolumn{5}{|l|}{ Working status during pregnancy } \\
\hline Employment & & & & Reference \\
\hline Freelance & 1.4287 & 3.7576 & 0.0526 & $4.173(0.984-17.694)$ \\
\hline Housewife/Unemployment & 0.5463 & 0.5702 & 0.4502 & $1.727(0.418-7.129)$ \\
\hline \multicolumn{5}{|l|}{ Delivery mode suggested by friends } \\
\hline No Suggestion & & & & Reference \\
\hline Natural Delivery & -0.6237 & 0.7062 & 0.4007 & $0.536(0.125-2.296)$ \\
\hline Cesarean Section & 3.6155 & 17.9693 & $<0.0001$ & $37.172(6.986-197.797)$ \\
\hline No Specific Suggestion & 0.3351 & 0.2475 & 0.6189 & $1.398(0.373-5.235)$ \\
\hline \multicolumn{5}{|l|}{ Age } \\
\hline$<20$ & 1.1 .307 & 1.3429 & 0.2465 & $3.098(0.458-20.968)$ \\
\hline $20-29$ & & & & Reference \\
\hline $30-35$ & 1.8381 & 7.9015 & 0.0049 & $6.285(1.745-22.641)$ \\
\hline \multicolumn{5}{|l|}{ Gestation-parturition history } \\
\hline No & & & & Reference \\
\hline Yes & 1.242 & 3.4176 & 0.0645 & $3.462(0.928-12.919)$ \\
\hline
\end{tabular}

reduce the rate of cesarean section [17-19]. The selective intention of cesarean section in pregnancy excluded medical indications factors such as large baby size and dystocia, which were mainly affected by social factors [20].

\section{Personal factors}

The selective intention of pregnant women's delivery mode is affected by multiple factors including physiology and psychology conditions. This study showed that the occupational status of pregnant women would affect the selective intention of cesarean section, and the unemployed or freelance would increase the risk of selective cesarean section. In addition, the lower the pregnant women's knowledge of delivery, the higher the risk of their selective intention to cesarean section. Many pregnant women blindly think that cesarean section is the safest mode of delivery, resulting in selective cesarean section. Therefore, delivery knowledge education for pregnant women can effectively reduce the rate of selective cesarean section. Moreover, no exercise in the late pregnancy could also increases the risk of selective cesarean section, because over weight would cause a huge newborn [21]. Thus pregnant women would worry about the risk of natural delivery, and the prenatal depression would also increase the probability of selective cesarean section. The early and middle pregnancies are the golden period for fetal development, while depression in pregnant women would not only affects fetal 
development, but also affects the belief for natural delivery. Moreover, negative emotions can amplify the pain of natural delivery and the negative effects of vaginal relaxation. Therefore, encouraging and caring about changes in the mental state of women during pregnancy could guide women with natural delivery conditions to choose natural delivery. The research results also indicated that too young or too old would increase the risk of pregnant women choosing cesarean section as a delivery mode, due to younger or older pregnant women will worry about whether their physical conditions are suitable for natural delivery. So encouraging pregnant women to give birth at an appropriate age can effectively reduce selective cesarean delivery. Besides, our research found that pregnant women with a reproductive history were more likely to choose cesarean section. However, with the "second child" policy in China, many families choose to reproduce one, but due to the increase in pregnant women's advanced age and many other pregnancy complications, the selective cesarean section rate of postpartum women has increased [22].

\section{Social factors}

Previous studies have shown that the rate of cesarean section in China was as high as $46.2 \%$. Among them, selective cesarean section with non-medical indications accounted for $11.7 \%$, becoming the highest in the world [23]. This study revealed that delivery mode suggested by family and friends were important factors affecting pregnant women's selective intention of cesarean section. Because many pregnant women lack childbirth experience, their choice of delivery mode would be more based on suggestions from family and friends, calling as "an experienced hand", with a one-sided understanding of delivery they usually blindly believe that cesarean section is a safe and reliable method, but do not know the adverse effects and consequences of cesarean section. Moreover, in mainland China, most of the elders believe in some traditional superstitions. They choose cesarean section in order to let the newborn be born on the auspicious day they believe, and some hold the view that cesarean section is conducive for the recovery of the pregnant woman's body and will not affect the sexual life of the couple. In addition, pregnant women's friends and family's description of the pain of natural delivery may reduce the confidence of them in natural delivery, just because they are afraid of pain and choose cesarean section as the delivery mode early. Therefore, relevant health institutions should carry out health education on delivery mode for pregnant women to let them know the basic process and precautions of different delivery modes, so as to improve their cognitive level of delivery knowledge. In addition, much attention should be paid to their family and friends' knowledge of delivery to eliminate the misunderstanding of delivery knowledge, and appropriately guide and control their suggestions, which can effectively reduce the selective intention of cesarean section with nonmedical indications.

\section{Gestation period}

Studies have shown that the weight and psychological status of pregnant women change with pregnancy, and prenatal screening and psychological intervention can reduce the prenatal depression of pregnant women [24]. The psychological state of pregnant women in the late pregnancy may decrease their fear of delivery, if constructive psychological intervention and family care have been taken, so cesarean section will not be chosen. Besides, our research also indicated that the age and gestation-parturition history of pregnant women in the late pregnancy were the important factors that affect their selective intention of cesarean section. With the change of pregnancy week, pregnant women may have a higher knowledge level of delivery. It is the objective conditions that affect pregnant women's choice of cesarean section in late pregnancy period, the physical condition of pregnant women can't support a natural delivery. Therefore, it can be seen that the selective intention of cesarean section of pregnant women in late pregnancy is significantly affected by the medical indications of cesarean section.

\section{Limitations}

This study has some limitations. We did not examine the experience of the "previous" vaginal delivery. Previous bad experience during vaginal delivery may increase the choice of cesarean section, whereas previous good experience will inevitably reduce it. We did not examine this effect in this study.

\section{Conflict of interest}

None

\section{Funding sources}

This work was funded by the National Natural Science Foundation of China (No.71, 573, 027).

\section{Acknowledgments}

The authors acknowledge the support of the Yubei Maternity and Child Healthcare Hospital, the Jiangjin Maternity and Child Healthcare Hospital, the Yunyang County Maternity and Child Healthcare Hospital, the Dianjiang People's Hospital and participants as well as the hard works of team members in the study.

\section{References}

1. Su Guo, Zhang Meiling, Zhang Ningzhi (2015) The choice of delivery mode of second pregnancy after cesarean section. J Bengbu Med Coll 40: 1063-1065.

2. Lumbiganon $P$, Laopaiboon $M$, Gulmezoglu AM, Souza JP, Taneepanichskul S, et al. (2010) Method of delivery and pregnancy outcomes in Asia: the WHO global survey on maternal and perinatal health 2007-08. Lancet 375: 490-499.

3. Dursun P,Yanik FB, Zeyneloglu HB, Baser E, Kuscu E, et al. (2011) Why women request cesarean section without medical indication? J Matern Fetal Neonatal Med 24: 1133-1137.

4. Huang Xin, Zhang Shaoling-Ling, Tan Hongzhuan, Lei Jun (2013) Analysis on determinants of cesarean section without medical indication. China Journal of Modern Medicine 20: 99-104.

5. Dong Wen, Lu Guiqiang, Liu Xiaojin (2015) Comparative analysis of the mode of second pregnancy and delivery after cesarean section. Hebei Medical Journal 1844-1845.

6. XV Haiyan, et al. (2013) Effect of antenatal clinic of midwifery nurse on delivery of old primipara. J Reproductive Med 22: 370-372.

7. Wang Na, Ding Yan (2012) Research status and Countermeasures of cesarean section in pregnant women without indication of cesarean section. Chin J Nurs 47: 751-754.

8. Zhou Huimin, Zhang Songtao, Hao Lixia, et al. (2011) Analysis of social factors and intervention in the increase of cesarean section rate. China Prac Med 6: 256-257.

9. Zhu Yanhuai (2010) The effect of health education during pregnancy on reducing the rate of cesarean section without medical indications. Practical Prev Med 17: 1236-1237.

10. Guo Sufang, Zhao Fengmin, Wu Kuangshi, CUI ying, WU jiuling, et al. (2005) Trends of caesarean section and related social and demographic factors in China from 1971 to 2003. Chinese J Perinat Med 3: 145-149. 
11. Shahoei R, Riji HM, Saeedi ZA (2011) 'Safe passage': pregnant Iranian Kurdish women's choice of childbirth method. J Adv Nurs 67: 2130-2138.

12. Zhang Ping, Shi Huimin, Wang Wenjuan (2019) The impact of midwifery out-patient delivery education on the choice of delivery methods and outcomes of pregnant women. Chinese J Coal Industry Med 22: 105-108.

13. Zhang ZJ, et al. (2005) Behavioral Medicine Scale Manual. $2^{\text {nd }}$ Edition, Chinese Medical Electronic Audio and Video Publishing House, Beijing, China, 214-215, 267-268.

14. Pan YL, Gao LL, Jin XH (2004) A Comparative Study on Psychological Stress of Pregnant Women and Their Spouses. Guangdong Medical Journal 10: 1209-1210.

15. Smilkstein G, Ashworth C, Montano D (1982) Validity and reliability of the family APGAR as a test of family function. J Fam Pract 15 : 303-311.

16. Shiwen S, Junqin L, Huilian B, Xiaoyan Y (2018) Survey and Analysis on Present Situation of Social Support and Family Care for Pregnant Women with Fetal Abnormality. J Nursing and Rehabilitation 17: 1619.

17. Barber EL, Lundsberg LS, Belanger K, Pettker CM, Funai EF, et al
(2011) Indications contributing to the increasing cesarean delivery rate. Obstet Gynecol 118: 29-38.

18. Betrán AP, Merialdi M, Lauer JA, Bing-Shun W, Thomas J, et al. (2007) Rates of caesarean section: analysis of global, regional and national estimates. Paediatr Perinat Epidemiol 21: 98-113.

19. Stjernholm YV, Petersson K, Eneroth E (2010) Changed indications for cesarean sections. Acta Obstet Gynecol Scand 89: 49-53.

20. Yu Cheng, Zhang Qiushi (2018) A survey and Study on the cognition of delivery mode of pregnant and lying in women in tertiary hospital. Health vocational education 36: 123-124.

21. Wang Qing, Li Guanghui (2016) Associations between third trimester gestational weight gain, maternal lipid profile \& prepregnancy body mass index and neonatal birth weight in pregnant women with normal glucose tolerance. Chinese J family plan 2016: 818-823.

22. Su Yan, et al (2019) Correlation analysis between the two child policy and the increase of cesarean section rate. Famous doctor 2019: 63

23. Zhang J, Liu Y, Meikle S, Zheng J, Sun W, et al. (2008) Cesarean delivery on maternal request in southeast China. Obstet Gynecol 111: 1077-1082.

24. CCM Ng, FM Lai, GSH Yeo (2004) Assessment of maternal anxiety levels before and after amniocentesis. Singapore Med J 45: 370-374. 